\title{
Reduction based Histogram Equalization Technique for Image Enhancement
}

\author{
Rupashri Barik, Tanusree Saha, Supriyo Chatterjee
}

\begin{abstract}
Here the proposed scheme mainly emphasizes the procedure of histogram equalization of images in more efficient way. Histogram equalization is required for image enhancement. Histogram spreads or flattens the histogram of an image and due to this the pixels with lower intensity values appear darker and the pixels with higher intensity values appear lighter. So the contrast of the input image is improved. For human interpretation various techniques of image enhancement have been widely used in different applications areas of image processing as the subjective quality of images is mainly important.
\end{abstract}

Keywords: Histogram, Histogram equalization, Probability Density Function, Cumulative Distribution Function.

\section{INTRODUCTION}

Enhancement of an image is a very important feature of digital image processing for providing better contrast. Enhanced image produces more details of input image as compare to non enhanced image. Image enhancement has various applications area. This technique is mostly used to enhance images like captured in remote sensing, medical images etc.Histogram [1] is an very important feature related to enhancing an image. An image histogram doesn't provide only a overall view on some image statistics, but it is also useful for predicting the intensity characteristic and general appearence of an image. The image is mostly a dark image when its histogram is condensed on the lower parts of the intensity level. Otherwise, an image is to be considered as a bright image as the histogram is condensed on the higher values of the intensity scale.Hence here is the requirement of equalizing the histogram of an image. Histogram equalization [1] designates the different intensity values of the pixels present in the input image so that uniform distributions of different intensities are contained in the output image. So the contrast is improved. The aim of equalizing a histogram of an image is mainly to get a uniform histogram for the same image. Histogram equalization produces a new histogram which intensity

Revised Manuscript Received on September 22, 2019.

Rupashri Barik,Assistant Professor, Department of Information Technology, JIS College of Engineering Kalyani, Nadia, 741235, W. B., India,barikrupashri@gmail.com

Tanusree Saha,Assistant Professor, Department of Information Technology, JIS College of Engineering Kalyani, Nadia, 741235, W. B., India ,tanusreesah@gmail.com

Supriyo Chatterjee,Student, Department of Information Technology, JIS College of Engineering,Kalyani, Nadia, 741235, W. B. India,sahebwap@gmail.com. values are closely grouped to be spreaded out into a flatten or equalized histogram. It can be achieved by having one transformation function which can be considered to be a Cumulative Distribution Function (CDF) [2] of a given Probability Density Function (PDF)[2] of the gray levels for the given image. PDF gives the probability of every gray level values in the image. Basically it provides the count or frequency for each gray value. $\mathrm{CDF}$ is the function which computes the cumulative sum for every gray values which are computed by PDF. It actually sums the previous one.

\section{IMAGE ENHANCEMENT}

\section{Image Representation}

An image can be defined as a two dimensional light intensity function $f(x, y)$, where $(x, y)$ represents spatial coordinates of the image and at any point $(x, y)$ the value of $f$ is proportional to the gray levels or brightness of the image at that particular point. In other words, the digital form of an image is an image $f(x, y)$ which has been discretized with the help of brightness and spatial coordinates. Pixels are the picture elements of such a matrix or of a digital array. So a digital image [1] can be represented using a 2D array or by a matrix. The representation of an digital image is as follows.

\section{Histogram}

Histogram of an image is the graphical presentation of the tonal value or gray value distribution of a digital image. It plots the count of pixels for each gray value or tonal value. The whole tonal distribution of an image can be judged by observing the histogram for a specific image.

The histogram [1] of the digital image with gray levels or intensity levels in the range $[0, \mathrm{~L}-1]$ can be represented as the discrete function $\mathrm{h}(\mathrm{rk})=\mathrm{nk}$

Here, rk represents the k-th intensity value,nk represents the count of pixels in the image with intensity rk.

Histogram is also represented as $\mathrm{p}(\mathrm{rk})=\mathrm{nk} / \mathrm{MN}$, where $\mathrm{M}$ and $\mathrm{N}$ represents the no. of rows and columns respectively in the image matrix. Histograms of the images may be represented graphically simply plotting $\mathrm{h}(\mathrm{rk})=\mathrm{nk}$ compared to rk.

Histogram [3] also can be considered as a graphical representation of pixel distribution over the luminous intensity. It is the estimation of the probability distribution for a continuous variable. 


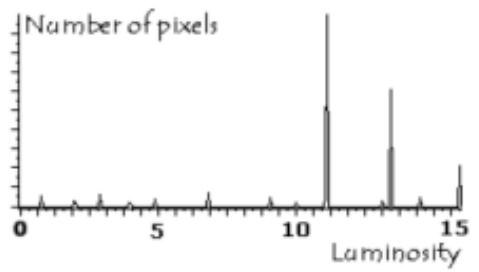

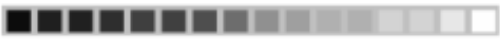

Fig1: Representation of Histogram

Image histograms are present in many modern digital cameras. These in-build tools help the photographers to understand the tonal distribution of captured image. It also can be used to find whether the details of the image has been lost to black-out shadows or blown-out highlights. While using a raw image format then it is less useful due to the dynamic range of the displayed image. It may only be an approximation to that in the raw image.

\section{Histogram equalization}

Histogram equalization [4] is an effective method for processing images in order to fine- tune the contrast of an image by altering the distribution of the intensity for the histogram. The main purpose of this technique is to get a linear trend to the cumulative probability function connected to the image. The perspective is to build a transformation so that the gray value in the output is uniformly distributed. Histogram equalization [5][6] is the point process which again distributes the intensity distributions of images in such an order to get an flatten or equalized histogram for the image. For equalizing the histogram of an image firstly the probability density function (PDF) to be calculated for all the pixels in the image, next the cumulative distributive function $(\mathrm{CDF})$ are to be calculated. After that the calculated $\mathrm{CDF}$ values are to be multiplied with one less than the gray values. Finally, the newly obtained gray level values should be mapped with the number of pixels

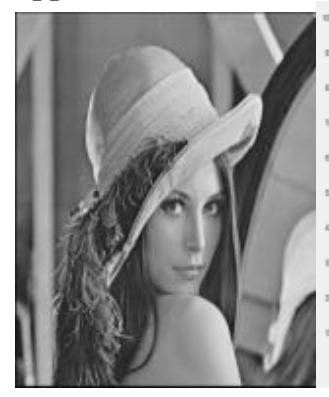

Fig 2a: Original Image

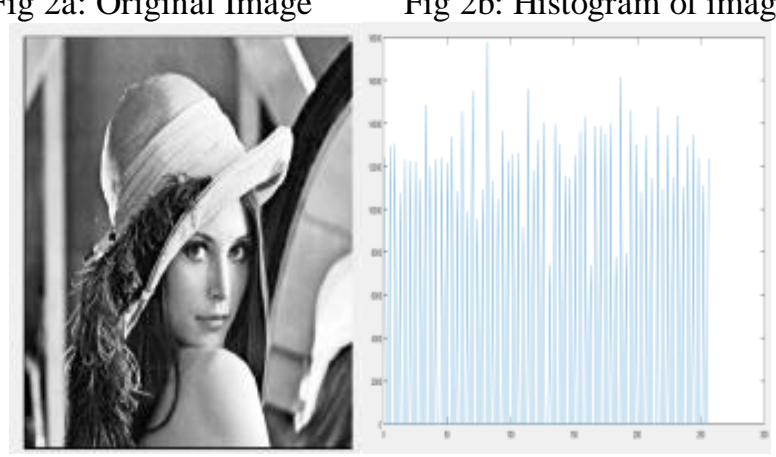

Fig 2c: Image after Equalization Fig 2d: Histogram after Equalization
Histogram equalization method is very much useful for enhancing the contrast of the image. It is not obligatory that contrast will always be increased at any means. Some situations may be there where histogram equalization [7][8] can be worse. In that aspect the contrast may decreased sometimes. It fulfils this by effectully spreading out the most frequent intensity values, i.e. expading out the intensity range of the image. This allows for areas of lower local contrast to gain a higher contrast. Traditional histogram equalization [9][10] method is very simple. The main disadvantage of this technique is that if there are gray values those are physically far apart from each other in the image, then this method fails. A disadvantage of the method is that it is indiscriminate. It may increase the contrast of background noise, while decreasing the usable signal.

\section{ALGORITHM}

In this algorithm of histogram equalization a new method has been developed for equalization of histogram, rather it can be stated that it is an improved version of conventional histogram equalization technique. In this proposed algorithm at first the image has been taken as input. Then the gray value matrix of the input image has been generated, after that for each of the row the minimum gray value is to be found. Next, the minimum gray values of each row are being subtracted from each of the elements present in that corresponding row including itself. After this subtraction the probability of occurrences of each pixel in the image have to be calculated. Then a new image matrix will be generated. Now for this new matrix the occurrence of each of the pixel value need to be counted, there after the cumulative sum should be generated. After that the ratio of occurrence of each of the pixel to its corresponding cumulative sum is calculated and that value should be multiplied with the biggest pixel value available in the matrix and the multiplication result is needed to be rounded off and those round off values are the new gray values of the image matrix. After that a new matrix will be created by replacing the old values with newly calculated values for each of the pixel values and there after a new matrix will be created. This image matrix will be the equalized histogram image of the original raw image. Now for this new matrix the occurrence of each of the pixel value need to be counted, there after the cumulative sum should be generated. After that the ratio of occurrence of each of the pixel to its corresponding cumulative sum is calculated and that value should be multiplied with the biggest pixel value available in the matrix and the multiplication result is needed to be rounded off and those round off values are the new gray values of the image matrix. After that a new matrix will be created by replacing the old values with newly calculated values for each of the pixel values and there after a new matrix will be created. This image matrix will be the equalized histogram image of the original raw image. 


\section{Proposed Algorithm At a glance}

Step 1: Read the image

Step 2: Find out the minimum pixel values from each row of the image matrix.

Step 3: Subtract the minimum pixel values of each row from the corresponding rows of image matrix.

Step 4: Count the number of occurrences of each pixel in the image matrix.

Step 5: Find the cumulative distributive function.

Step 6: Find the ratio of occurrence of pixel to the cumulative sum.

Step 7: Multiply the maximum grey value of pixel with each of the ratio.

Step 8: That multiplication result is the equalized pixel value.

Step 9: Map the new gray values with the old gray values.

Step 10: Get the equalized image.

Step 11: Exit

Fig 3: Proposed Algorithm for Histogram Equalization The above algorithm shows how to equalize the histogram of an image to enhance the quality of an image. As in conventional method [1][13] if the grey values are far apart from each other so the equalized image doesn't give proper contrast, concentrating on this point here that limitation has been tried to reduce to get an enhanced image. The proposed algorithm is not suitable to for the images which are having zero pixel values in each row of the image matrix. In this case the image obtained from histogram equalization [11][12] will be quite similar to the original image.

\section{RESULTS \& DISCUSSIONS}
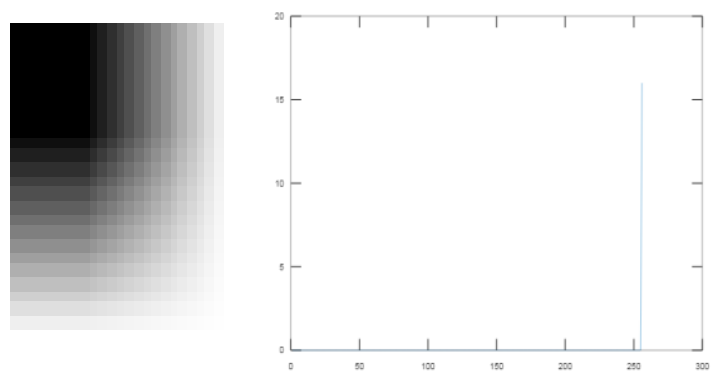

FIG 4A: ORIGINAL IMAGE FIG 4B: HISTOGRAM OF IMAGE

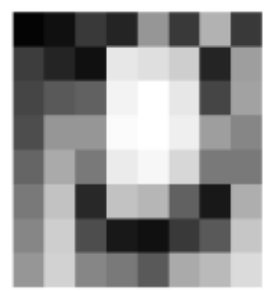

Fig 4c: Image after Equalization

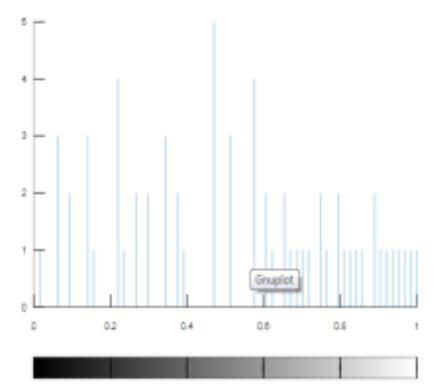

Fig 4d: Histogram after Equalization using Traditional Method

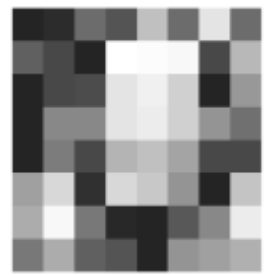

Fig 4e: Image after Equalization

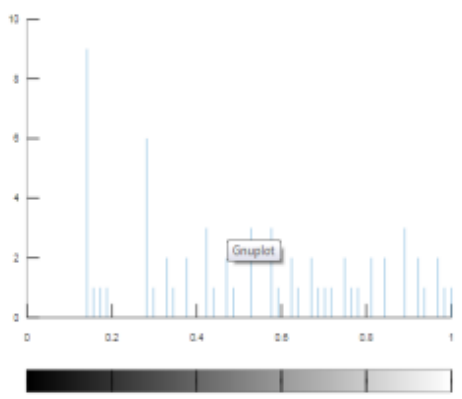

Fig 4f: Histogram after Equalization using Proposed Algorithm

After implementing the proposed algorithm in MATLAB [14][15], the above results have been obtained. Here one random image has been taken as an input image. Fig. $4 \mathrm{a}$ is the input image and Fig. $4 \mathrm{~b}$ is the corresponding histogram of the input image. Fig.4c and Fig. $4 d$ are the diagram of the equalized image and the corresponding histogram respectively. These two diagrams have been obtained after applying traditional histogram equalization technique. Finally applying reduction based histogram equalization technique Fig. 4e and Fig. 4f have been obtained which are representing the equalized image and the corresponding histogram respectively. The difference between Fig. 4d and Fig. $4 \mathrm{f}$ is very clear. Here the observation is that, in Fig. $4 \mathrm{f}$ the histogram is more expanded and the peaks values are quite flatten than Fig. $4 \mathrm{~d}$ and that is the aim of histogram equalization. It also can be observed that various gray levels are present in the equalized image comparison to the original image. input image and Fig. $4 \mathrm{~b}$ is the corresponding histogram of the input image. Fig. $4 \mathrm{c}$ and Fig. $4 \mathrm{~d}$ are the diagram of the equalized image and the corresponding histogram respectively. These two diagrams have been obtained after applying traditional histogram equalization technique. Finally applying reduction based histogram equalization technique Fig. $4 \mathrm{e}$ and Fig. $4 \mathrm{f}$ have been obtained which are representing the equalized image and the corresponding histogram respectively. The difference between Fig. 4d and Fig. 4f is very clear. Here the observation is that, in Fig. 4f the histogram is more 
expanded and the peaks values are quite flatten than Fig. 4d and that is the aim of histogram equalization. It also can be observed that various gray levels are present in the equalized image comparison to the original image.

\section{CONCLUSIONS}

Equalization of histogram is a very much effective technique for improving the contrast of an image. The proposed algorithm overcomes the shortcoming of gray values those are far apart but it can't handle zero gray values in the image matrix in proper way. Nowadays digital image processing has a great impact to our society in respect to the science and research. Medical sciences, defence issues, space systems, etc. are very much influenced by several image processing techniques and for this purposes image enhancement is highly required so histogram equalization is also essential to improve the contrast of the images.

\section{REFERENCES}

1. R. C. Gonzalez and R. E. Woods, Digital image processing, 2nd edition, Prentice-Hall of India, 2004.

2. Pooja Mishra, Mr. KhomLal Sinha, "Different Approaches of Image Enhancement", International Journal of Research in Advent Technology, Vol.2, No.8, August2014.

3. Harpreet Kaur1, Neelofar Sohi, "A Study for Applications of Histogram in Image Enhancement", The International Journal of Engineering and Science (IJES), Volume 6, Issue 6, PP 59-63, 2017.

4. http://www.mathworks.com/products/demos/\#

5. http://www.math.siu.edu/MATLAB/tutorials.html 